\title{
A RARE CASE OF GAS GANGRENE OF ABDOMINAL WALL IN PREGNANCY
}

KEY WORDS: gas gangrene, pregnancy, Cl. perfringens, IUFD, abortion

\section{Dr. Himani Baxy* Dr. Vijay Y. Kalyankar Dr. Shrinivas Gadappa Dr. Megha Chauhan} Junior Resident, Dept. of Obstetrics and Gynaecology, Govt. Medical College and Hospital, Aurangabad. *Corresponding Author

Associate Professor, Dept. of Obstetrics and Gynaecology, Govt. Medical College and Hospital, Aurangabad.

Gas gangrene in pregnancy is a rare occurrence although associated with high mortality. We report the case of a patient
who developed gas gangrene of abdominal wall post-partum. A 30-year-old G3P2L2 with 6 months of amenorrhea, a
neglected ANC with no antenatal visits, a known case of quadriparesis, delivered a preterm male baby. Having a
contusion of $20 * 10 \mathrm{~cm}$ size on lower abdomen, a blister formed over the same post-partum which had to be debrided. She
was treated vigorously with antibiotics and tetanus antitoxin; and appropriate supportive management. Patient's
condition worsened after $36 \mathrm{hrs}$ and $46 \mathrm{hrs}$ after admission she went into cardiac arrest. And such we encountered a case
in 2019 in modern-day obstetrics, of gas gangrene complicating pregnancy, costing life to both the new-born and
parturient. The importance of early suspicion and diagnosis followed by prompt, vigorous treatment cannot be
emphasized more.

\section{INTRODUCTION:}

Gas gangrene complicating pregnancy is a rare entity encountered in present-day obstetrics; but mortality due to gas gangrene remains high. Many obstetricians don't even come across such a case. It is both unexpected and interesting when such cases do occur even today and they are of sufficient value to warrant reporting.

Sadusk and Manahan (1941) found that 8.7 per cent of pregnant women had Cl. perfringens on vaginal culture(1), although the organism was not necessarily a permanent inhabitant.

Of all the cases of gas gangrene in pregnancy reported, about two thirds have occurred following an abortion, while cases occurring late in pregnancy had a dead fetus in utero and difficult deliveries which involved tissue damage and necrosis. Gas gangrene in pregnancy can affect multiple organs. It can cause extensive hemorrhagic endomyometritis warranting hysterectomy. The infection may become generalized and affect other organs. Septicemia with hepatic and renal failure, pulmonary intra-alveolar edema can occur in short duration.

\section{CASE HISTORY:}

The patient was a 30 -year-old woman, a third gravida with previous two normal deliveries, residing in a rural area and illiterate. She was referred from a sub-district hospital with bleeding per vaginum since four hours as '24 weeks of pregnancy with placenta previa in bleeding phase and a known case of 'quadriparesis' due to atlantoaxial dislocation.' There was no history of bleeding disorders, nausea, vomiting, headache and blurring of vision. Bedridden for three years, unaware of her pregnancy, she was a neglected ANC, receiving Tab. Gabapentin 300mg 1/2 tablet twice-a-day for three months.

\section{EXAMINATION:}

The patient was vitally stable with normal blood pressure. Per abdominally uterine size was $22-24$ week. An old contusion of size $20 * 10 \mathrm{~cm}$ size on lower abdomen was noted. On per speculum examination, cervix was fully dilated, bag of membranes forming and bulging. No active bleeding per vaginum. Patient didn't have any blood investigation and ultrasonography reports with her. She received antibiotics and tetanus immunisation. Patient was kept for spontaneous delivery as she had no active bleeding. Patient delivered vaginally preterm male baby weighing $800 \mathrm{~g}$ within $2 \mathrm{hr} 15$ min.

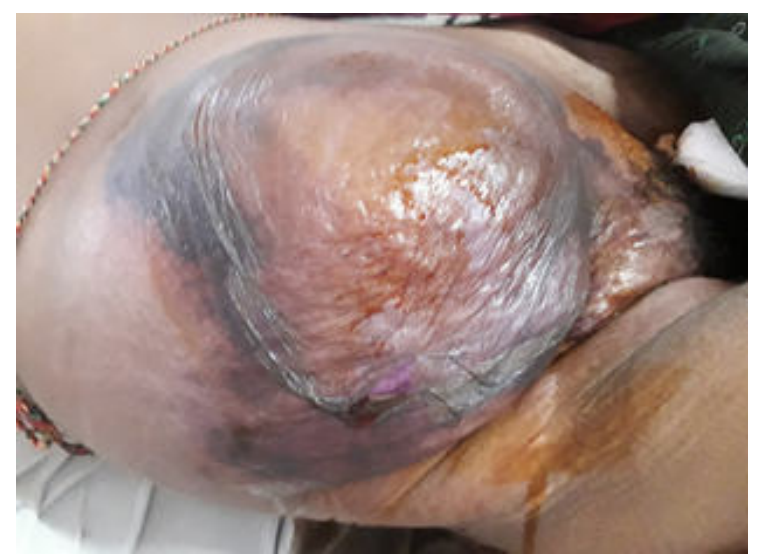

Figure no. 1: Contusion Of Size $2 * 10 \mathrm{~cm}$ Size On Lower Abdomen

Patient was stable till 11 hours. Then she developed a large blister on abdomen on lower part of $20 * 10 \mathrm{~cm}$ which was aspirated under all aseptic precautions from periphery and had foul smelling serous fluid. Abdominal wall cellulitis was suspected. Few hours later she had a skin patch of $20 * 10 \mathrm{~cm}$ present on lower abdominal wall. Blood pressure had fallen to $70 / 50 \mathrm{mmHg}$ and patient was hypoglycemic, febrile with tachycardia disproportionate to temperature. Patient was taken for emergency debridement in operation theatre. All possible necrotic gangrenous skin and subcutaneous tissue cut and removed. Underlying muscle with gangrenous changes present, with involvement of subcutaneous fat with subcutaneous gas pockets present. Necrotic gangrenous muscle tissue sample cut and sent for gas gangrene evaluation. Betadine-hydrogen peroxide-soaked pack kept. 


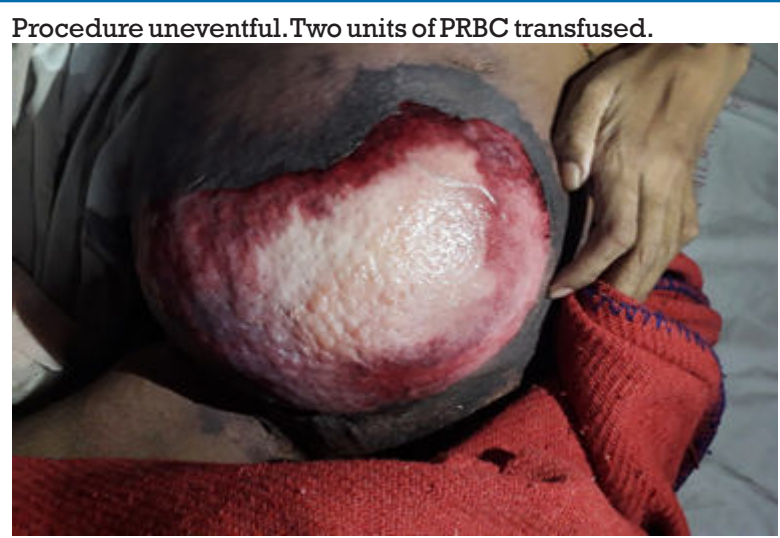

Figure no. 2: Lesion After Emergency Surgical Debridement

24 hours from admission, patient's condition deteriorated. She became drowsy and disoriented. Patient shifted to Surgical Intensive Care Unit (I.C.U.) on ventilation.

Now, about $36 \mathrm{hrs}$ from admission, in Surgical I.C.U., patient's condition was poor, conscious, on AMBU, with presence of 30 * $30 \mathrm{~cm}$ size ulcer over anterior abdominal wall. Slough and gangrenous necrotic muscle present. Patient was shifted to higher antibiotic- Inj. Piperacillin + Tazobactam 4.5g TDS and appropriate fluid and pain management was done.

After two hours, patient became unconscious, blood pressure not recordable. Patient was given steroids and ionotropes, antibiotics continued.

Patient revived and kept on AMBU ventilation. Patient was given Inj. Atropine lcc iv stat and was kept on noradrenaline support with addition of Inj. Ceftriaxone $1 \mathrm{~g}$ TDS. At about 46 hours after admission, patient went into sudden cardiac arrest and declared dead.

\section{DISCUSSION:}

Jones DH found that Clostridium perfringens is ubiquitous and is found vaginally in ca. $1-10 \%$ of healthy women and usually does not cause a serious infection.(1) the presence of injured and devitalised tissue creating an anoxic nidus for growth of these anaerobic organisms is what leads to activation of spores of the same. Comorbid conditions such as diabetes mellitus, uremia, low immunity and gross anemia and virulence of organisms predispose to infection. As in one study it was found that only 5 percent of the strains of $\mathrm{Cl}$. perfringens cultured from the genital tract were virulent.(1)

Kirkpatrick CJ, Werdehausen $\mathrm{K}$, Jaeger J, Breining $\mathrm{H}$. in their study concluded that Clostridial infections after delivery are more common with artificial or early spontaneous rupture of membranes, operative delivery, fetal death, prolonged labor with many vaginal examinations, retained placental tissue and the presence of other virulent organisms.(2) In our patient, none of these conditions existed except for the presence of a contusion of unknown mode i.e. devitalised tissue and her not have taken Inj. Tetanus toxoid during pregnancy as she had paid no antenatal visits. (3)

Jones DH and WD R. described features in a case of gas gangrene such as pain, tachycardia disproportionate to fever and a lucid mental characterising gas gangrene. Patients have a toxic look but are conscious till the end, as was in our case too. Their colour varies according to the stage of infectionthey are very pale at the beginning almost ashen grey; later a combination of livid cyanosis and jaundice induces a coppery or mahogany colour. Haemoglobin levels fall rapidly, moderate leucocytosis, spherocytosis and in severe cases methemoglobinemia is present. There may be profound haemolysis. Patients complain of shivering attacks and the skin is cold and sweating- pulse rate is disproportionately raised as compared to temperature- which may be normal, sub-normal or slightly elevated. The respiration is increased.(1,2) In our case, patient had slight fever with profound tachycardia and increased respiratory rate.

On examination, there is exquisite tenderness and crepitation at times can be felt through the wall of distended abdomen. Our patient had tenderness but no crepitations, as a large blister was present which was aspirated.

According to study conducted by $\mathrm{KH}$ S., a vaginal discharge, always present, consists of serous fluid and altered blood.(4) The urinary output is reduced and may stop altogether. Urine color is burgundy or port-wine since it contains methemoglobin and oxyhemoglobin. Vaginal and cervical swabs gram positive, encapsulated, rod shaped bacillus, but treatment must be commenced without waiting for bacteriological confirmation. X-ray- gas may be seen in affected region. In our case, urine and blood culture were sterile. Vaginal swab showed Klebsiella pneumoniae culture. Aspirated fluid from blister in microscopy revealed gram positive bacilli of large, square-built linear form, with round ends.

Treatment of gas gangrene needs to be prompt and vigorous. Its aim is to destroy the invading organisms and neutralize toxins. At the same time, supportive treatment to the vital organs damaged by toxins must be given. And the role of debridement in gynecology is less defined $(2,5)$. Montavon C, Krause E, Holzgreve W, Hösli I. Uteriner Gasbrand described a case of a 28-year female with previous one LSCS who had delivered by vacuum extraction and developed gas gangrene of uterus and adnexa was seen with unilateral extension to abdomen reaching as far as axilla cranially and thigh caudally. In this case surgical treatment by doing hysterectomy, necrosis removal and splitting of fascia followed by several debridements save the woman's life.(5)

Treatment with penicillin or other present-day antibiotics must be done.(2,5,6) Penicillin is not contraindicated in pregnancy and thus can be administered in an ANC. The role of antitoxins is less well established. If the patient is allergic to penicillin, she can be given alternative antibiotic such as erythromycin. In our case we used Inj. Piperacillin with Tazobactam as higher antibiotic and polyvalent antitoxin; along with supportive treatment in the form of correction of fluid and electrolyte imbalance.

\section{CONCLUSION:}

Even in the present era, we may get a case of gas gangrene in pregnancy. Clear sensorium with tachycardia out of proportion to the temperature, who is complaining of severe pain in the area involved which cannot be explained otherwise should raise suspicion. A neglected antenatal care with comorbid conditions predisposes the woman for catching infection. Emphasis must be on good obstetric care, identifying the risk factors and early and prompt detection of gas gangrene in pregnancy with vigorous treatment.

\section{REFERENCES}

1. Jones DH. GAS GANGRENE COMPLICATING PREGNANCY. BJOG An Int J Obstet Gynaecol. 1965 Oct;72(5):785-90.

2. Kirkpatrick CJ, Werdehausen K, Jaeger J, Breining H. Fatal clostridium perfringens infection after normal term pregnancy. Arch Gynecol. 1982 Mar;231(2):167-70.

3. WD R. Gas Gangrene Complicating Term Pregnancy. Obstet Gynecol 1960;15.

KH S. [On Puerperal Gas Gangrene].Zentralbl Gynakol. 1967;89(6).

5. Montavon C, Krause E, Holzgreve W, Hösli I. Uteriner Gasbrand mit Clostridium Perfringens Sepsis nach intrapartaler gedeckter Uterusruptur. (Uterine Gas Gangrene through Clostridium Perfringens Sepsis after Uterus Rupture Postpartum). Z Geburtshilfe Neonatol .2005 Oct;209(5):167-72.

6. GLW,OT B,WN J. GAS GANGRENE IN PREGNANCY. Ala J Med Sci. 1964;1 